\section{Susceptibility-Weighted MR Phase Imaging Can Demonstrate Retrograde Leptomeningeal Venous Drainage in Patients with Dural Arteriovenous Fistula}

We read with special interest the article of Mittal et al ${ }^{1}$ entitled "Susceptibility-Weighted Imaging: Technical Aspects and Clinical Applications, Part 2." The authors did a critical review of the most important clinical applications of susceptibility-weighted MR phase imaging (SWI). They emphasized the possible role of SWI for the evaluation of patients with traumatic brain injury, stroke, cerebrovascular disease, neurodegenerative disorders, multiple sclerosis, brain tumors, vascular malformations, and so forth. They demonstrated the usefulness of SWI in patients with dural sinus thrombosis because it can demonstrate venous stasis and collateral slow flow. Regarding the vascular malformations, Mittal et al mentioned the advantages of SWI, because cavernous malformations, developmental venous angiomas, and capillary telangiectasias have slow flow and can be less conspicuous or even missed by conventional MR imaging techniques. However, the role of SWI in cases of dural arteriovenous fistulas (DAVFs), another potential clinical application of this sequence, was not discussed in this article.

We report the case of a 71-year-old man with progressive dementia and a previous history of hemorrhagic infarcts. He underwent brain MR imaging, which showed areas of encephalomalacia with hemosiderin/ferritin deposits and mild microangiopathic changes. In addition to these findings, the SWI demonstrated severe dilation of the cerebral veins on the surface of the brain as well as deep in the brain (Fig 1). Due to these findings, the diagnostic hypothesis of DAVF with retrograde leptomeningeal venous drainage (RLVD) was suggested. Digital subtraction angiography was performed and confirmed the diagnosis of left transverse-sigmoid sinus DAVF with RLVD.
Intracranial DAVFs with RLVD can cause intraparenchymal and subarachnoid hemorrhage, focal neurologic deficits, seizures, and dementia. ${ }^{2}$ The presence of RLVD is the most important risk factor for a poor prognosis in patients with DAVF, and it can be well demonstrated with contrast-enhanced T1-weighted MR images. ${ }^{3}$ However, SWI not only better demonstrates the venous drainage impairment in these patients but also has the benefit of no intravenous contrast administration. Further studies should investigate the role of SWI for the evaluation of DAVF, but it is clear that this sequence can add valuable information for these patients.

\section{References}

1. Mittal S, Wu Z, Neelavalli J, et al. Susceptibility-weighted imaging: technical aspects and clinical applications, part 2. AJNR Am J Neuroradiol 2009;30:232-52

2. Kitajima M, Hirai T, Korogi Y, et al. Retrograde cortical and deep venous drainage in patients with intracranial dural arteriovenous fistulas: comparison of MR imaging and angiographic findings. AJNR Am J Neuroradiol 2005;26:1532-38

3. Lee SK, Willinsky RA, Montanera W, et al. MR imaging of dural arteriovenous fistulas draining into cerebellar cortical veins. AJNR Am J Neuroradiol 2003;24:1602-06

E.L. Gasparetto

Department of Radiology University Federal of Rio de Janeiro

C.E. Pires

University of Rio de Janeiro State R.C. Domingues

Clínica de Diagnóstico Por Imagem Rio de Janeiro, RJ

Brazil
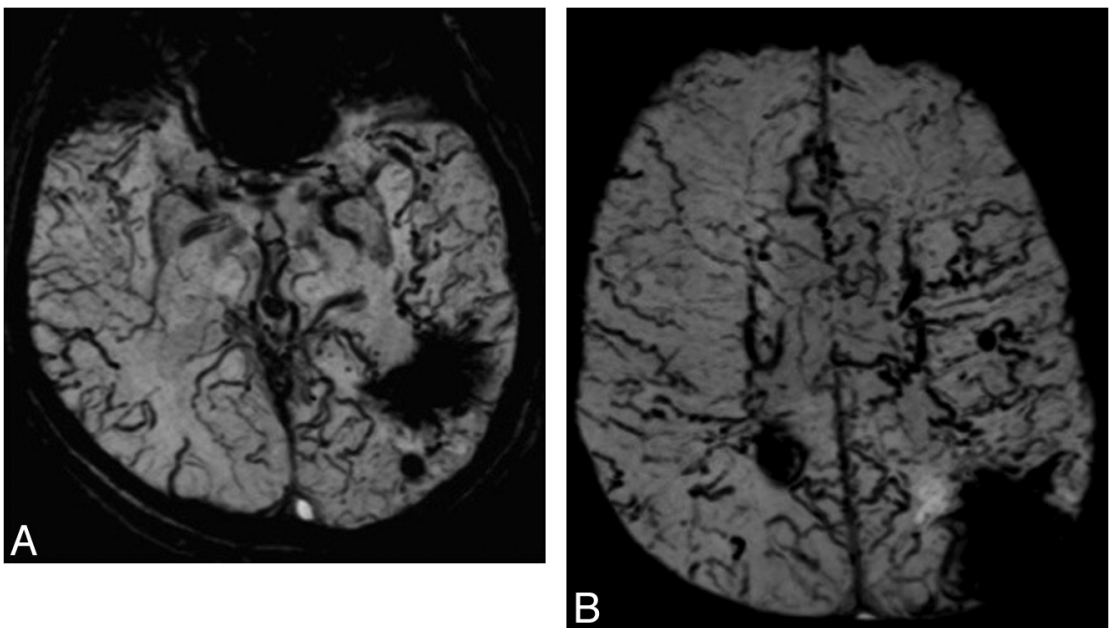

Fig 1. Axial SWI minimum-intensity-projection images show severe dilation of cortical and medullary veins in both hemispheres. In addition, old hemorrhagic infarcts are seen in the parietal lobes and left temporal lobe. 